\title{
Electron irradiation effects on the nucleation and growth of Au nanoparticles in silicon nitride membranes
}

\author{
M. M. Timm, Z. E. Fabrim, C. Marin, D. L. Baptista, and P. F. P. Fichtner
}

Citation: Journal of Applied Physics 122, 165301 (2017); doi: 10.1063/1.4998734

View online: https://doi.org/10.1063/1.4998734

View Table of Contents: http://aip.scitation.org/toc/jap/122/16

Published by the American Institute of Physics

\section{Articles you may be interested in}

Influence of incoherent twin boundaries on the electrical properties of $\beta-\mathrm{Ga}_{2} \mathrm{O}_{3}$ layers homoepitaxially grown by metal-organic vapor phase epitaxy

Journal of Applied Physics 122, 165701 (2017); 10.1063/1.4993748

Composite films of highly ordered Si nanowires embedded in $\mathrm{SiGe}_{0.3}$ for thermoelectric applications Journal of Applied Physics 122, 165302 (2017); 10.1063/1.4986355

High-temperature crystallized thin-film PZT on thin polyimide substrates

Journal of Applied Physics 122, 164103 (2017); 10.1063/1.4990052

Electric and magnetic properties of magnetic $(\mathrm{CoFeTaB})_{(100-x)} \mathrm{O}_{\mathrm{x}}$ films

Journal of Applied Physics 122, 165101 (2017); 10.1063/1.4998996

Modeling emission lag after photoexcitation

Journal of Applied Physics 122, 164501 (2017); 10.1063/1.5008366

Modeling quantum yield, emittance, and surface roughness effects from metallic photocathodes

Journal of Applied Physics 122, 165303 (2017); 10.1063/1.4996568

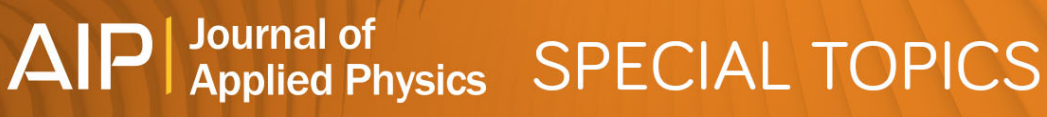

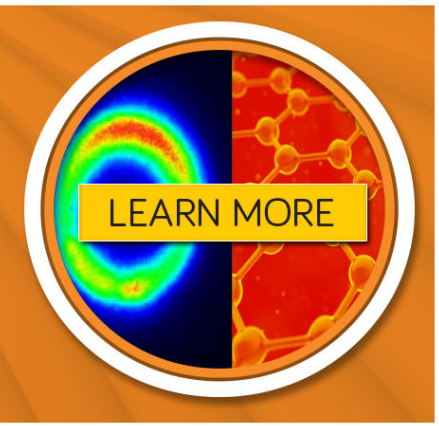




\title{
Electron irradiation effects on the nucleation and growth of Au nanoparticles in silicon nitride membranes
}

\author{
M. M. Timm, ${ }^{1,2}$ Z. E. Fabrim, ${ }^{2,3}$ C. Marin, ${ }^{1,2}$ D. L. Baptista, ${ }^{2}$ and P. F. P. Fichtner ${ }^{1,2,3,4}$ \\ ${ }^{1}$ Programa de Pós-Graduação em Ciência dos Materiais, Universidade Federal do Rio Grande do Sul, \\ Porto Alegre 91501-970, Brazil \\ ${ }^{2}$ Instituto de Física, Universidade Federal do Rio Grande do Sul, Porto Alegre 91501-970, Brazil \\ ${ }^{3}$ Programa de Pós-Graduação em Microeletrônica, Universidade Federal do Rio Grande do Sul, \\ Porto Alegre 91501-970, Brazil \\ ${ }^{4}$ Escola de Engenharia, Universidade Federal do Rio Grande do Sul, Porto Alegre 91501-970, Brazil
}

(Received 2 August 2017; accepted 30 September 2017; published online 23 October 2017)

\begin{abstract}
The formation of Au nanoparticles (NPs) in $\mathrm{Au}^{+}$ion-implanted silicon nitride thin films and membranes was investigated as a function of post-implantation thermal treatments or room temperature electron irradiation at energies of $80,120,160$, and $200 \mathrm{keV}$. The samples were characterized by Rutherford Backscattering Spectrometry and Transmission Electron Microscopy. High-temperature thermal annealing $\left(1100^{\circ} \mathrm{C}, 1 \mathrm{~h}\right)$ resulted in the formation of $\mathrm{Au}$ particles with a mean diameter of $\approx 1.3 \mathrm{~nm}$. In comparison, room-temperature electron irradiation at energies from 80 to $200 \mathrm{keV}$ caused the formation of larger Au particles according to two growth regimes. The first regime is characterized by a slow growth rate and occurs inside the silicon nitride membrane. The second regime presents a fast growth rate and starts when Au atoms become exposed to the back free surface of the membrane. Realistic binary electron-atom elastic collision cross-sections were used to analyze the observed nanoparticle growth and membrane sputtering phenomena. The results obtained demonstrate that binary electron-atom elastic collisions can account for the microstructure modifications if the critical displacement energies for the sputtering of $\mathrm{N}$ and $\mathrm{Si}$ atoms are around $14 \pm 3 \mathrm{eV}$, and the displacement energy for surface located Au atoms is approximately $1.25 \pm 0.2 \mathrm{eV}$. Irradiation experiments using focused electron probes demonstrate that the process provides fine control of nanoparticle formation, resulting in well-defined sizes and locations. Published by AIP Publishing.

https://doi.org/10.1063/1.4998734
\end{abstract}

\section{INTRODUCTION}

Nano-sized objects have a large fraction of their constituent atoms located at the interface with the surrounding medium. In addition, their dimensions can be comparable with the characteristic length of their electronic and/or lattice excitations. This combined situation allows for sizedependent physical and chemical properties. For example, in the case of metallic nanoparticles (NPs), their sizes determine the melting temperature, ${ }^{1}$ latent heat,${ }^{1,2}$ electrical properties, ${ }^{3,4}$ HOMO-LUMO energy gap value, ${ }^{5}$ and plasmonic resonance frequencies, ${ }^{6}$ among other properties influencing distinct applications. ${ }^{7}$ The synthesis method of the nanoobject system determines how the object sizes can be controlled. For surface or bulk processes based on solid state precipitate reactions, particle sizes are frequently tailored by their nucleation and growth rates, which can be thermally controlled either in situ or upon post-synthesis thermal annealing treatments.

Nano-object systems are intrinsically outside of thermodynamic equilibrium conditions. With increasing temperature, the particles tend to coarsen, with the reduction of the system free interface energy being the major driving force of the process. In most cases, the thermal robustness of the system or of the individual particles strongly depends on the particle-medium interface properties. For example, with decreasing sizes, Au and Sn nanoparticles (NPs) present a reduction of their melting temperature $T_{m}$ to approximately one half of the bulk value at the particle diameter $d \approx 2 \mathrm{~nm}$. This behavior can be well explained by distinct melting theories, where the difference between the particle interface energy of the solid and the liquid phase is an important parameter. ${ }^{8,9}$ However, in the case of free standing Sn particles, or even for $\mathrm{Sn}$ and $\mathrm{Pb}$ particles embedded in silica, the depression behavior can be reversed, leading to an increase of the $T_{m}$ values up to 1.5 times larger than the bulk values for particle diameters $d<1.5 \mathrm{~nm} .^{10,11}$ This discrepancy was explained by considering that, for the size range $d<1.5$ $\mathrm{nm}$, the $\mathrm{Sn}$ and $\mathrm{Pb}$ atoms tend to form covalent bonds (instead of the metallic ones) and a specific atomic arrangement without dangling bonds in the case of free standing particles or fully coherent interfaces in the case of embedded particles. Consequently, the particle cohesion energy is enhanced and the particle-medium interface energy is reduced, thus increasing the thermal toughness of the individual particles. ${ }^{10-12}$ Nanoparticle coarsening occurs by means of thermally induced atomic displacements via two distinct mechanisms. The first mechanism is based on the absorption and resolution of solute atoms by the particles. In this case, after a short transient stage, the solute field tends to be maintained by the equilibration of the chemical potential between each individual particle and the matrix. The equilibrium condition, typically described by the Gibbs-Thomson principle, ${ }^{13}$ depends on the particle-matrix interface energy 
and on the particle diameter. These parameters determine whether a given particle will shrink or grow with respect to the neighboring ones, leading to an overall competitive growth process known as Ostwald ripening. ${ }^{14,15}$ The second mechanism comprises the atomic displacement of particlematrix interface atoms, leading to the migration of the hole particle, following a Brownian-like process, where coarsening occurs when two particles collide. This mechanism is mostly effective for liquid or gaseous particles and is commonly known as "migration and coalescence."

Distinct from the thermally induced atomic displacements, irradiation by energetic particles (electrons, ions, or neutrons) may produce atomic displacements at much lower temperatures. If the irradiation process does not affect the chemical potential conditions of the system, then the basic thermodynamic driving forces are not affected; however, the coarsening behavior can be significantly accelerated and therefore become operative at lower temperatures. The synergy provided by electron irradiation induced migration and coalescence has been recently documented for the case of $\mathrm{Pb}$ NPs in silica submitted to in situ irradiations at $1100^{\circ} \mathrm{C} .{ }^{16}$ Under such extreme conditions, impressive particle migration speeds were reported. In the present contribution, we study the formation and growth of Au NPs in ion-implanted silicon nitride membranes, exploring the effects of room temperature electron irradiations. In comparison to the effects of high-temperature thermal annealing, the results obtained demonstrate that Au NPs in silicon nitride form a thermally tough system that can be tailored via electron irradiation for either bulk or surface located particles. The results are discussed in terms of binary electron-atom elastic collision effects. This approach provides guidelines to understanding the microstructure modifications of the target material and of the atomic migration processes causing the nucleation and growth of the particles. The electron irradiation process is also explored as a tool to provide wellcontrolled sizes and particle locations.

\section{EXPERIMENTAL}

In the present experiments, silicon nitride Silson ${ }^{\circledR}$ selfstanding $100 \mathrm{~nm}$ thick membranes supported by a $200 \mu \mathrm{m}$ thick silicon frame were implanted with $\mathrm{Au}^{+}$ions using a $500 \mathrm{kV}$ HVEE ion implanter (Ion Implantation Laboratory, Physics Institute, Federal University of Rio Grande do SulUFRGS). The implantations were performed at room temperature with an energy of $E_{i}=80 \mathrm{keV}$ to a fluence of $\Phi_{i}=1 \times$ $10^{16} \mathrm{~cm}^{-2}$. Larger pieces of $200 \mathrm{~nm}$ thick $\mathrm{Si}_{3} \mathrm{~N}_{4}$ films deposited over silicon wafers were also implanted, either using the same conditions of the self-standing membranes or with $E_{i}=430 \mathrm{keV}$ and $\Phi_{i}=1 \times 10^{16} \mathrm{~cm}^{-2}$ to test the microstructural evolution upon high vacuum thermal annealing at distinct temperatures up to $1100^{\circ} \mathrm{C}$ for $2 \mathrm{~h}$. Au concentrationdepth profiles from the thin film samples were measured by Rutherford Backscattering Spectrometry (RBS), using a $1.2 \mathrm{MeV} \mathrm{He}^{+}$beam from a $3 \mathrm{MeV}$ HVEE Tandem accelerator from the same laboratory. These samples were also investigated by transmission electron microscopy (TEM) using cross-section specimens prepared by ion milling. The TEM observations were performed using a JEM 2010 microscope (Center for Microscopy and Microanalysis, UFRGS) equipped with a $\mathrm{LaB}_{6}$ filament. The same microscope was also used to irradiate the self-standing membranes (plan view geometry). The electron irradiations were performed at energies $E_{e}$ of 80 , 120,160 , and $200 \mathrm{keV}$, with a magnification of $6 \times 10^{5}$ times using a slightly focused beam converging onto an area of approximately $250 \mathrm{~nm}$ in diameter. The irradiated regions observed by TEM correspond to an inner circle of approximately $150 \mathrm{~nm}$ in diameter, where the electron irradiation beam current is approximately uniform and achieves values up to $1 \mathrm{~A} \mathrm{~cm}^{-2}$. The irradiation current was measured on the small screen and was previously calibrated using the Faraday cup from a low-background Gatan holder. During the irradiation, the beam current was kept constant. At regular times, the irradiation current was reduced to proceed with microstructural characterization and then increased again to accumulate a new irradiation dose in the same region. Additional irradiation experiments to test for the formation of localized nanoparticles were conducted at $300 \mathrm{kV}$ using a probe corrected Titan 80-300TM microscope from the Brazilian National Bureau of Standards (INMETRO).

\section{RESULTS}

Figure 1(a) shows the RBS measurements of as-implanted, aged $\left(200^{\circ} \mathrm{C}, 100 \mathrm{~h}\right)$ and annealed $\left(1100^{\circ} \mathrm{C}, 1 \mathrm{~h}\right)$ samples from the $\mathrm{Si}_{3} \mathrm{~N}_{4}$ films implanted with $\mathrm{Au}\left(E_{i}=430 \mathrm{keV}\right.$ and $\Phi_{i}=1 \times 10^{16} \mathrm{~cm}^{-2}$ ). The Au signal was converted into a concentration-depth profile using the surface energy approximation. ${ }^{17}$ The Au profile remained unchanged after the annealing, indicating that no significant depth redistribution of $\mathrm{Au}$ atoms occurred. High-resolution Z-contrast TEM micrographs obtained from cross-section specimens illustrate the local arrangement of the Au atoms in aged [Fig. 1(b)] and annealed [Fig. 1(c)] samples. In this imaging mode, the Au atoms appear as white dots, which are clearly distinguishable from the darker background. There was no significant Au agglomeration in the aged sample. Upon annealing at $1100^{\circ} \mathrm{C}$ for $1 \mathrm{~h}$, however, most of the Au atoms agglomerated into small clusters characterized by a size distribution with a mean diameter of $d_{m}$ $\approx 1.3 \mathrm{~nm}$ and a standard deviation of $\Delta d \approx 0.23 \mathrm{~nm}$.

To characterize the $\mathrm{Au}$ concentration-depth profile in the membranes used on the electron irradiation experiments, silicon nitride films were implanted with Au using the same parameters of the membrane implantation $\left(E_{i}=80 \mathrm{keV}\right.$ and $\Phi_{i}=1 \times 10^{16} \mathrm{~cm}^{-2}$ ). Figure 2(a) shows the as-implanted Au concentration-depth profile measured by RBS. The dashdotted curve represents the estimated profile resulting from the deconvolution of the detector resolution and the energy straggling from the He beam. The profile is characterized by a mean depth of $R_{p} \approx 22 \mathrm{~nm}$ and a standard deviation of $\Delta R_{p}$ $\approx 5 \mathrm{~nm}$ (corrected profile). The depth scale of the plot extends to the thickness of the membranes, and the arrows indicate the direction of the electron beam during the irradiation experiments. Figure 2(b) shows a bright-field phase-contrast TEM image (underfocus) of an as-implanted sample. In this contrast mode, the darker features represent regions containing a larger mass density. These features suggest that 

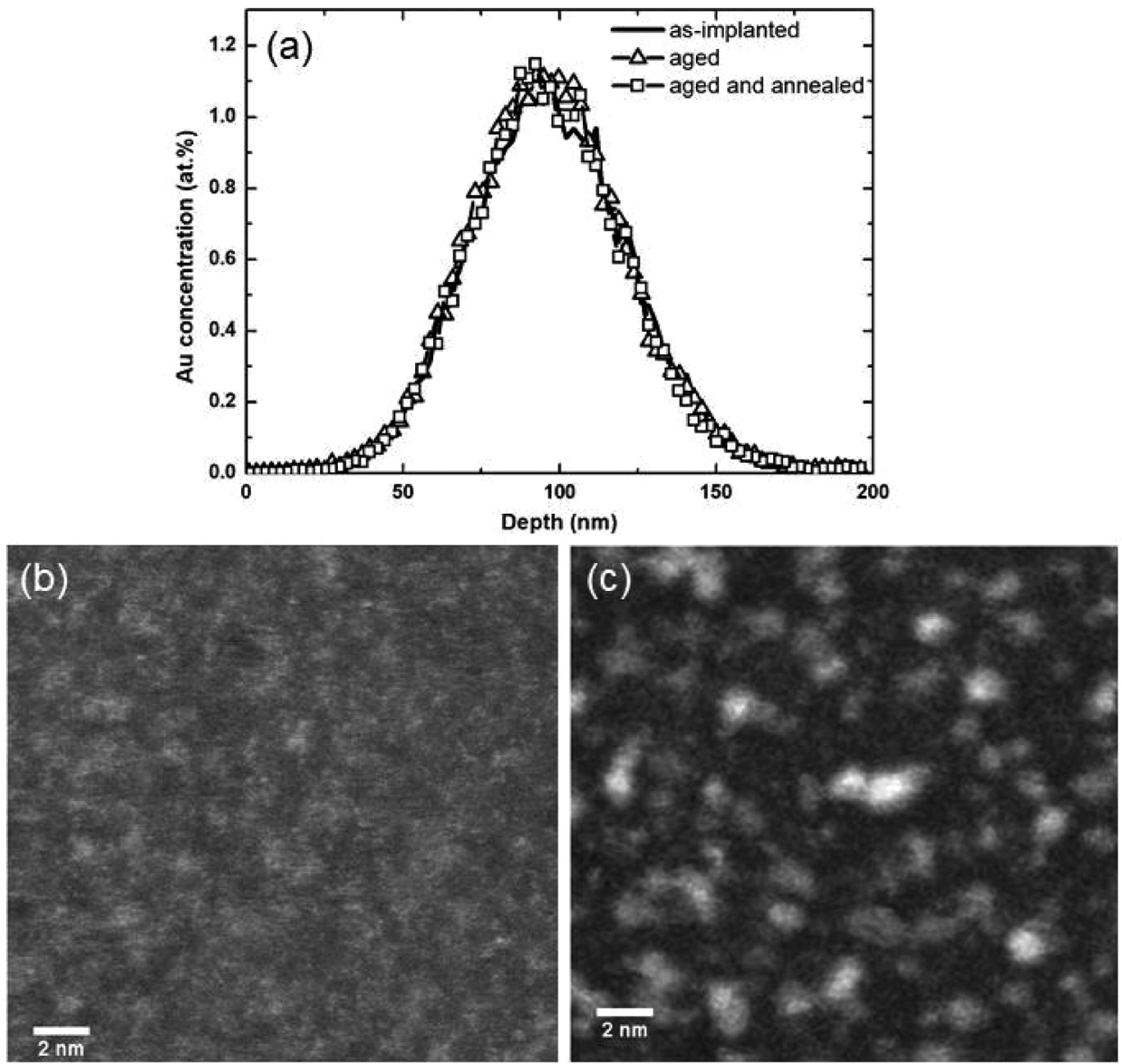

FIG. 1. (a) Concentration-depth profiles of the implanted $\mathrm{Au}$ in asimplanted, aged $\left(200^{\circ} \mathrm{C}, 100 \mathrm{~h}\right)$ and annealed $\left(1100^{\circ} \mathrm{C}, 1 \mathrm{~h}\right)$ samples. (b) High-resolution Z-contrast TEM micrograph from the aged sample. (c) Highresolution Z-contrast TEM micrograph from the annealed sample. small Au clusters were formed during the implantation process, which was very probable due to the higher atomic concentration of $\mathrm{Au}$ achieved by implantation (Au peak concentration $\approx 4.7$ at. $\%$ ) compared to the samples used in the annealing experiments shown in Fig. 1 (Au peak concentration $\approx 1.3$ at. $\%$ ).

The electron irradiation effects on the formation of $\mathrm{Au}$ agglomerates are illustrated in Fig. 3 for the case of an irradiation at $200 \mathrm{keV}$ in the irradiation direction 1 . The figure shows TEM micrographs illustrating the growth of $\mathrm{Au}$ agglomerates from samples irradiated to fluences $\Phi_{e}$ from 2.8 to $7.2 \times 10^{5} \mathrm{C} \mathrm{cm}^{-2}$. The diffraction contrast variation appearing in the image of some larger particles [see, e.g., Fig. 3(d)] reveals the formation of twin crystals. Figure 4(a) shows a low-magnification TEM image that presents an overall view of an irradiated area. The change in the background contrast of the irradiated region as compared to the surrounding non-irradiated areas suggests that, concomitant with the growth of the particles, the thickness of the sample within the irradiated region was locally reduced. Figure 4(b) shows a SAD pattern obtained from a sample irradiated to $\Phi_{e}=7.16 \times 10^{5} \mathrm{C} \mathrm{cm}^{-2}$. This pattern matches the face-centered-cubic structure of the Au crystal lattice, as identified by the diffraction rings from the (111), (200), (220), and (311) crystalline planes. Similar results were obtained for samples irradiated in the irradiation direction 2, demonstrating that the larger particles can be characterized as Au crystals. In the following, all Au agglomerates will be referred as
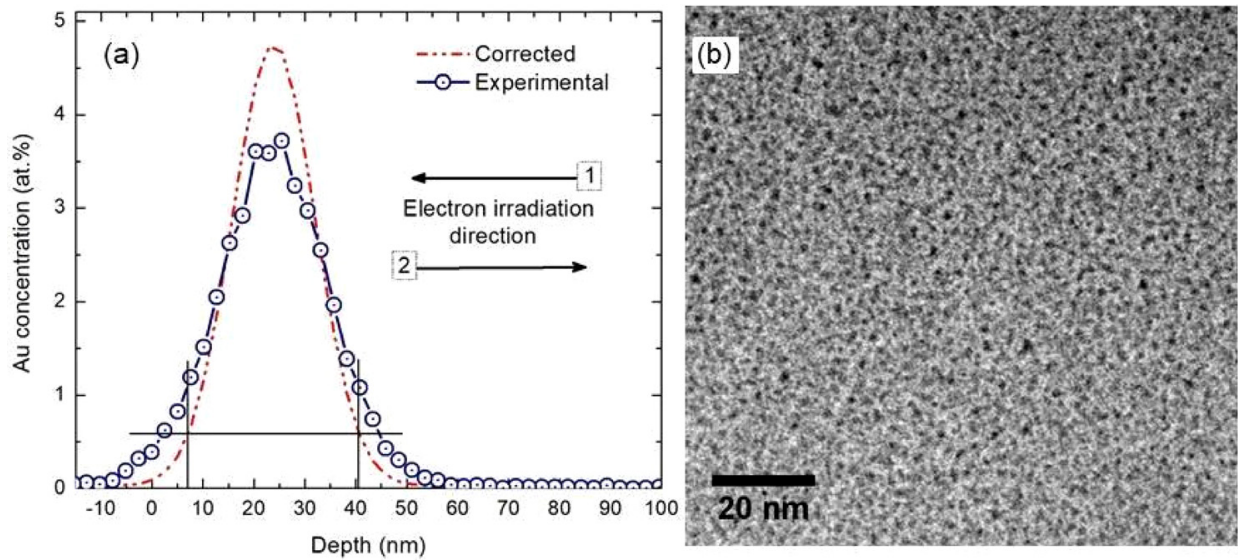

FIG. 2. (a) RBS measurement of the concentration-depth profile of $80 \mathrm{keV}$ implanted $\mathrm{Au}$ atoms in a $100 \mathrm{~nm}$ thick $\mathrm{Si}_{3} \mathrm{~N}_{4}$ film. The arrow indicates the ion implantation and the electron irradiation direction of the experiments using the membranes. (b) Bright-field (underfocus) plan-view TEM micrograph from the as-implanted sample. 

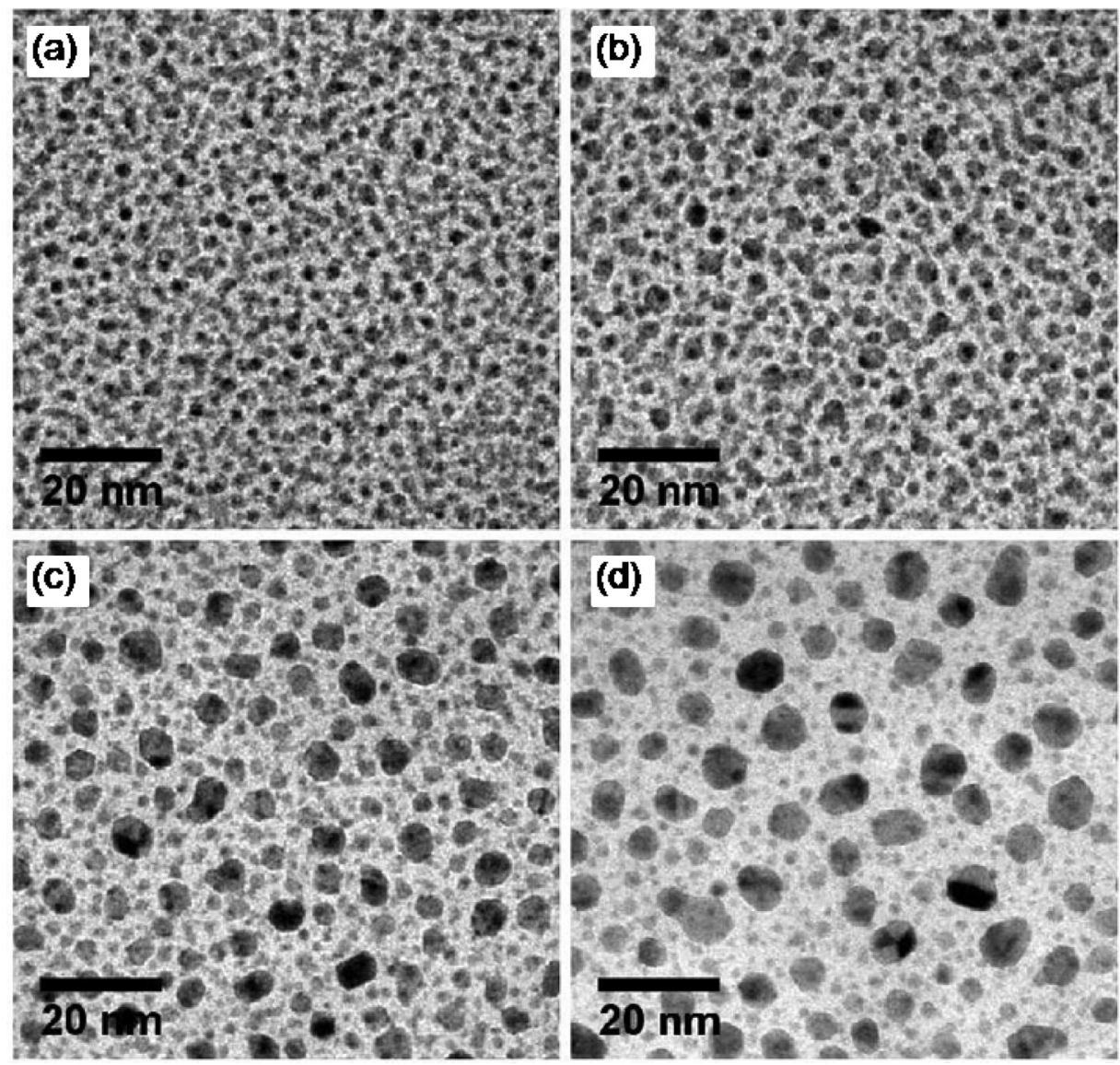

FIG. 3. TEM micrographs from a sample irradiated at $E_{e}=200 \mathrm{keV}$ for fluences $\Phi_{e}$ of (a) $2.81 \times 10^{5} \mathrm{C} \mathrm{cm}^{-2}$, (b) $3.99 \times 10^{5} \mathrm{C} \mathrm{cm}^{-2}$, (c) $5.58 \times 10^{5} \mathrm{C}$ $\mathrm{cm}^{-2}$, and (d) $7.16 \times 10^{5} \mathrm{C} \mathrm{cm}^{-2}$.
Au nanoparticles (NPs). Figure 5 shows the evolution of the mean diameter $\left(d_{m}\right)$ of Au NP size distributions for samples irradiated at $200 \mathrm{keV}$, comparing the results obtained for both irradiation directions. Figure 6 shows the $d_{m}$ behavior for irradiation experiments performed using the irradiation direction 1 with beam energies of 80, 120, 160, and $200 \mathrm{keV}$. The error bars in these figures correspond to the measurement error estimated in $\mp 0.3 \mathrm{~nm}$, combined with the standard error of the mean $\left(\sigma_{d_{m}}=\sigma / \sqrt{N}\right.$, being $\sigma$ the standard deviation and $N$ the number of particles in the particle size distribution). The overall results from the irradiation experiments were analyzed in terms of the evolution of $d_{m}$ (obtained from ensembles containing at least 150 particles) as a function of the electron irradiation fluence, energy, and irradiation direction.

\section{DISCUSSION}

The evolution of mean particle diameters $d_{m}$ (symbols) presents two distinct growth regimes for each irradiation direction (Fig. 5) or irradiation energy (Fig. 6). The first regime occurs for lower fluences and is characterized by a relatively small $d_{m}$ growth rate. The second regime starts after a given inflection fluence $\Phi_{e}^{i}$ where the particles demonstrate a faster growth. The continuous lines in both figures represent a linear fit for each growth regimen. The fits were
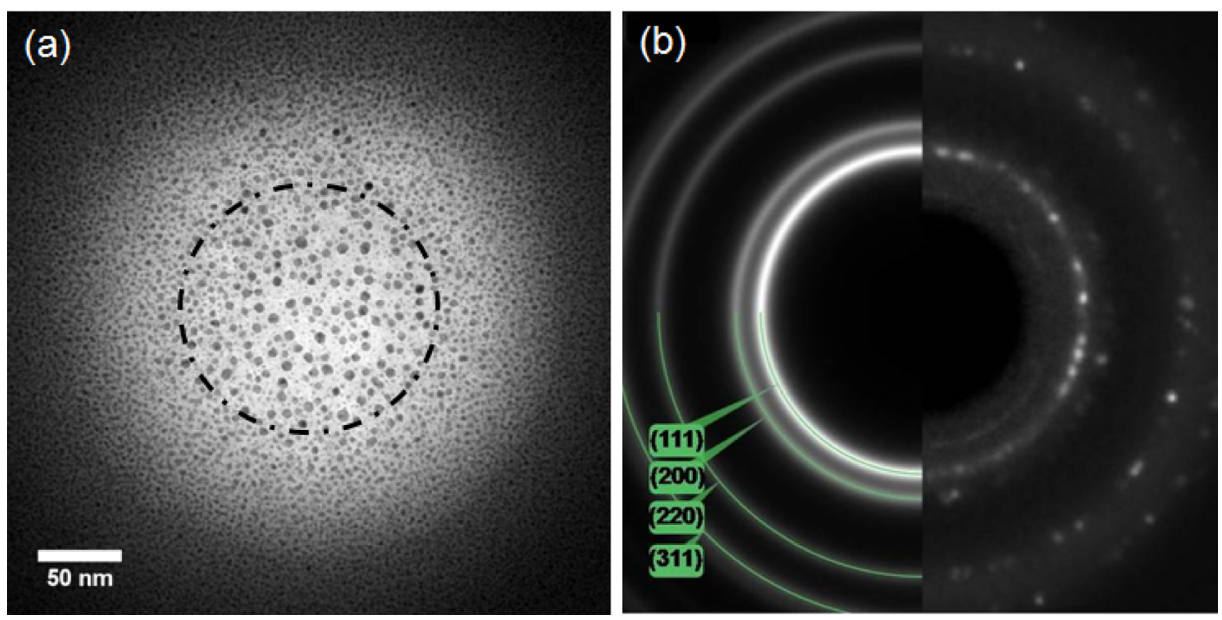

FIG. 4. (a) Low-magnification TEM micrograph illustrating the overall irradiation area $\left(E_{e}=120 \mathrm{keV}\right.$ and $\left.\Phi_{e}=7.92 \times 10^{5} \mathrm{Ccm}^{-2}\right)$. The irradiated area becomes brighter as a result of the local reduction in thickness. The circle with a diameter of $\approx 150 \mathrm{~nm}$ represents the region used to evaluate the particle size distribution. (b) SAD pattern from a set of particles (sample irradiated at $E_{e}=200 \mathrm{keV}$ to a fluence $\Phi_{e}=7.16$ $\left.\times 10^{5} \mathrm{C} \mathrm{cm}^{-2}\right)$. 


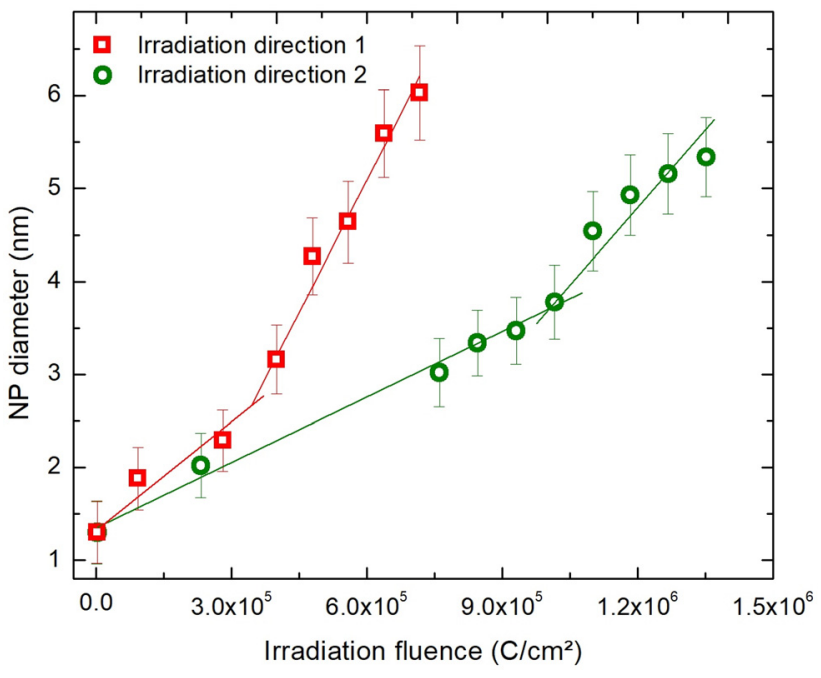

FIG. 5. Mean nanoparticle diameter $d_{m}$ as a function of the irradiation fluence $\Phi_{e}$ for experiments conducted at $200 \mathrm{keV}$ comparing irradiation directions 1 and 2 .

performed to determine the position of $\Phi_{e}^{i}$. The dotted lines in Fig. 6 represent model calculations to be further discussed below.

To discuss the results summarized in Figs. 5 and 6, we make the following assumptions: (i) radiolysis induced atomic displacements can be neglected since the NP growth increases with the irradiation beam energy, as opposed to the behavior of radiolysis cross-sections, ${ }^{18,19}$ (ii) electroninduced heating effects in the NPs can be neglected because of the large mass of the Au atoms and the small size of the particles; ${ }^{20}$ and (iii) heating effects in the silicon nitride membrane can be neglected since our heating experiments (see Fig. 1) demonstrate that, for annealing at $200{ }^{\circ} \mathrm{C}$ for $100 \mathrm{~h}$, no significant agglomeration could be observed. Furthermore, for annealing at $1100^{\circ} \mathrm{C}$ for $1 \mathrm{~h}$, no significant atomic redistribution in depth occurred; instead, only a local thermally induced agglomeration occurred that rendered $d_{m} \approx 1.3 \mathrm{~nm}$, which is quite similar to the initial values

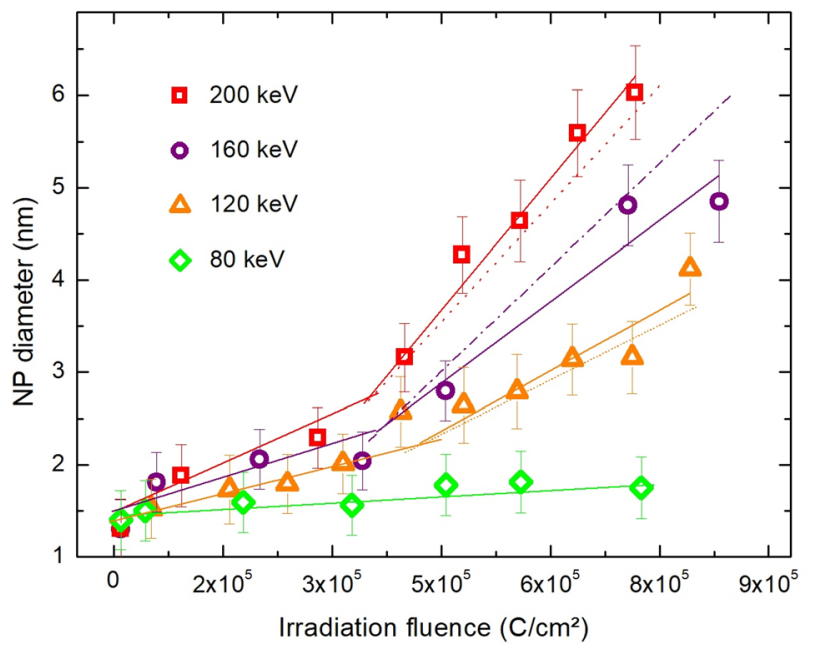

FIG. 6. Mean nanoparticle diameter $d_{m}$ as a function of the irradiation fluence $\Phi_{e}$ for experiments conducted at electron energies $E_{e}$ of 80,120,160, and $200 \mathrm{keV}$ for irradiation direction 1 . The dispersion bars in each data point correspond to the standard deviation from the particle size distributions. shown in Figs. 5 and 6. Hence, we may discuss the microstructure modifications observed in the present work in terms of binary electron-atom elastic collision processes with the $\mathrm{N}$, $\mathrm{Si}$, or $\mathrm{Au}$ atoms, either considering direct knock-on atomic displacements or considering that a displaced atom may also cause the displacement of a neighboring atom. The atomic displacement probability is described via elastic displacement cross-sections $\sigma$, where the key parameters are the electron beam energy $E_{e}$, the atomic number $\mathrm{Z}$, and atomic mass $\mathrm{A}$ of the target atoms, and the threshold energy $E_{d}$ necessary to cause a displacement of a bound atom. For thin membranes, we can also neglect the inelastic energy losses of the electrons and assume that $\sigma$ will not depend on the depth location of the atoms inside the sample. However, atoms located at the sample surfaces are less bound and should present smaller $E_{d}$ values compared to the bulk located ones. ${ }^{21}$ As a consequence, two displacement events should be considered: those occurring inside the sample that will cause local atomic rearrangements and/or migration and those occurring at the sample surface that will cause local atomic rearrangements, atomic migration, or sputtering of surface located $\mathrm{N}$ and $\mathrm{Si}$ atoms, leading to a local reduction of the membrane thickness. The sputtering process is considered to occur preferentially at the electron exit side of the membrane. $^{22,23}$

The two growth regimes outlined by the data in Figs. 5 and 6 can be discussed in terms of a simple phenomenological model. Concomitant with the formation of Au clusters observed in the first regime, the membrane thickness is preferentially reduced from the back surface (i.e., the electron exit side). This process continues until the $\mathrm{Au}$ atoms and clusters become exposed at the back surface, which typifies the inflection point $\Phi_{e}^{i}$ where the fast growth regime starts. The Au atoms exposed at the electron beam exit surface can be easily displaced, migrating along the surface, but not being sputtered. This process leads to a faster agglomeration, resulting in the formation of the larger particles. The fast growth process is sustained by a continuous supply of $\mathrm{Au}$ atoms as the sample thinning proceeds over the depth region containing the $\mathrm{Au}$ atoms. Figure 7 depicts the basic steps of the proposed phenomenological model. To test its consistency, we will analyze whether the membrane thinning process can be described in terms of electron induced sputtering of $\mathrm{N}$ and $\mathrm{Si}$ atoms from the electron beam exit surface, thus providing an explanation for the inflection point positions shown in Figs. 5 and 6.

To evaluate the sputtering rate $R$ for the silicon nitride membrane, we use a Bragg's rule approach ${ }^{17}$ to express the sputtering rate from a compound material in terms of the expression for monatomic materials provided by Crozier et al. ${ }^{22}$ The reduction in sample thickness caused by the sputtering process is given by the product R.t, which is defined as follows:

$$
R . t=\left(\frac{J . t}{e}\right)\left(\frac{u}{\rho}\right)^{1 / 3}\left[\frac{3}{7} \sigma_{S i} A_{S i}^{\frac{1}{3}}+\frac{4}{7} \sigma_{N} \mathrm{~A}_{N}^{\frac{1}{3}}\right] .
$$

In this expression, $J$ is the beam current; $t$ is the irradiation time; $e$ denotes the electric charge; $\sigma_{S i}$ and $\sigma_{N}$ denote the 


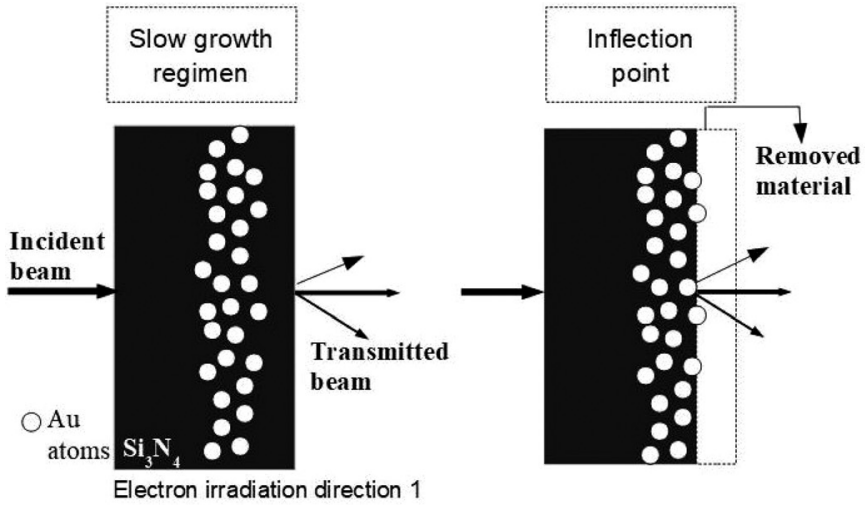

elastic displacement cross-sections for $\mathrm{Si}$ and $\mathrm{N}$ atoms, respectively; the factors $3 / 7$ and $4 / 7$ denote the membrane stoichiometry; $u$ is the atomic mass unit; $\mathrm{A}_{\mathrm{Si}}$ and $\mathrm{A}_{\mathrm{N}}$ are the atomic masses of $\mathrm{Si}$ and $\mathrm{N}$, respectively; and $\rho$ is the atomic density of the material. The product $J$.t corresponds to the experimental irradiation fluence $\Phi_{e}$, as shown in the x-axis of Fig. 5. Figure 2 suggests that the depth of the sputtered material, until the Au atoms are exposed to the interface, corresponds to a thickness of about $\approx 5$ to $10 \mathrm{~nm}$ for irradiation direction 1 and about 55 to $65 \mathrm{~nm}$ for irradiation direction 2 . The uncertainties in the thickness of the sputtered material represent the uncertainties in the membrane thickness. The amount of the sputtered material should be produced by the fluence $\Phi_{e}^{i}$, corresponding to the inflection point position for each beam direction or energy cases (see Figs. 5 and 6). The only unknown parameters in Eq. (1) are the displacement cross-sections $\sigma_{S i}$ and $\sigma_{N}$, which can be estimated using the McKinley-Feshbach approach expressed as follows: ${ }^{24}$

$$
\begin{aligned}
\sigma^{M F}= & B Z^{2} 4 \pi a_{0}^{2} R^{2} X+2 \pi \alpha \beta \sqrt{X}-\left(\beta^{2}+\pi \alpha \beta\right) \ln (X) \\
& -(1+2 \pi \alpha \beta) .
\end{aligned}
$$

In Eq. (2), $\beta=v / c$ ( $v$ is the speed of the electrons and $c$ is the speed of the light in vacuum), $B=\left(1-\beta^{2}\right) /\left(m_{0}^{2} c^{4} \beta^{4}\right)$, $m_{0} c^{2}=511 \mathrm{keV}, \alpha=Z / 137, R=13.6 \mathrm{eV}$ (Rydberg constant), $a_{0}=52.9 \mathrm{pm}$ (Bohr's radius), and $X=E_{\max } / E_{d}$, where $E_{\text {max }}=2 E_{0}\left(E_{0}+2 m_{0} c^{2}\right) / M c^{2}, \mathrm{~m}_{\mathbf{0}}$ is the electron rest mass, and $M$ is the atom mass. Figure 8 shows $\sigma_{N}^{M F}$ and $\sigma_{S i}^{M F}$ plots for various beam energies $E_{e}$ and displacement energies $E_{d}$.

Hence, considering $J . t \approx \Phi_{e}^{i}$ and $\rho=3.2 \mathrm{~g} \mathrm{~cm}^{-3}$, Eq. (1) renders distinct $R$.t values depending on the choice of the displacement energies $E_{d}$ for $\mathrm{N}$ and $\mathrm{Si}$. The results listed in Table I were obtained considering the uncertainties in the $\Phi_{e}^{i}$ values (estimated in about $\pm 5 \%$ ) and on the membrane thickness. Therefore, the obtained $E_{d}$ values also present uncertainties as quoted in the table. We assume $E_{d}^{S i}=E_{d}^{N}$,

TABLE I. Sputtered depth R.t calculated using Eq. (1).

\begin{tabular}{lccc}
\hline \hline$E_{e}(\mathrm{keV})$ & $\Phi_{e}^{i}\left(\mathrm{c} / \mathrm{cm}^{2}\right)$ & $E_{d}(\mathrm{eV})$ & R.t $(\mathrm{nm})$ \\
\hline 120 & $4.2 \pm 0.510^{5}$ & $13.5 \pm 3$ & $5-10$ \\
160 & $3.6 \pm 0.510^{5}$ & $14.5 \pm 3$ & $5-10$ \\
200 & $3.4 \pm 0.510^{5}$ & $16 \pm 3$ & $5-10$ \\
200 (direction 2) & $1.0 \pm 0.210^{6}$ & $11.5 \pm 2$ & $55-65$ \\
\hline \hline
\end{tabular}

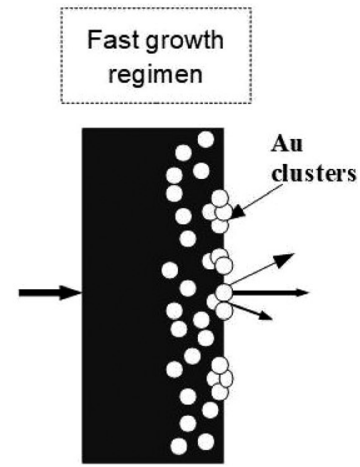

FIG. 7. Schematic representation of the sputtering process and its influence on the NP growth regimes. implying that the sputtering process is rate controlled by the removal of $\mathrm{N}$ atoms, thus reducing the bonding coordination of the Si atom. ${ }^{25}$ The concept of weakening the atomic bonding state to reduce the displacement threshold energy has also been explored to explain the sputtering of $\mathrm{N}$ atoms from $\mathrm{BN}$ nanotubes ${ }^{26}$ or damage formation via vacancy induced displacement in electron irradiated $\mathrm{InN} .^{27}$

Within the experimental errors and uncertainties, the results shown in Table I indicate that displacement energies in the range $E_{d} \approx 14 \pm 3 \mathrm{eV}$ consistently support, for all irradiation directions and beam energies, the concept that the inflection point represents the irradiation fluence where the $\mathrm{Au}$ atoms become exposed to a free surface, giving rise to the fast NP growth regime. According to Egerton et al. ${ }^{28}$ the threshold displacement energy for sputtering should be scaled in terms of the sublimation energy as $E_{d} \approx E_{\text {sub }}$ or $E_{d} \approx \frac{5}{3} E_{\text {sub }}$. Using the atomic $\mathrm{Si}-\mathrm{N}$ binding energy of $\approx 4.88 \mathrm{eV},{ }^{29}$ we may estimate sublimation energies $E_{\text {sub }}^{S i}$ $\approx 19.5 \mathrm{eV}$ and $E_{\text {sub }}^{N} \approx 14.6 \mathrm{eV}$, considering fully coordinated $\mathrm{Si}$ and $\mathrm{N}$ atoms in the silicon nitride structure. Under the assumption that the sputtering process is rate controlled by the removal of $\mathrm{N}$ atoms, our results are quite consistent with $E_{s u b}^{N} \approx 14.6 \mathrm{eV}$. In contrast, $E_{s u b}^{S i} \approx 4.7 \mathrm{eV}$ and $E_{s u b}^{N} \approx$ $2 \mathrm{eV}$ values are used for ion beam induced sputtering calculations by Ziegler and Biersack ${ }^{30}$ Furthermore, $E_{d}$ values between 20 and $25 \mathrm{eV}$ were estimated by Howitt et al. ${ }^{25}$ on the basis of electron beam hole drilling experiments in silicon nitride. Figure 8 suggests that, for $E_{d} \geq 20 \mathrm{eV}$, the $\sigma_{N}^{M F}$ values are rather small, and the $\sigma_{S i}^{M F}$ values are negligible for electron beam energies $E_{b} \leq 160 \mathrm{keV}$, which is in contradiction to the present experimental results.

Hereafter, we will discuss the growth of the Au NP based on the empirically determined inflection point fluences $\Phi_{e}^{i}$. According to our phenomenological model, the observed growth behavior of the Au NPs can be considered as a bulk phenomenon for electron beam fluences $\Phi_{e}<\Phi_{e}^{i}$ and as a surface phenomenon for $\Phi_{e} \geq \Phi_{e}^{i}$. In the bulk, since silicon nitride does not present a dense packed structure, atomic displacements induced by elastic collisions require a threshold displacement energy that can be estimated in terms of the sum of the individual atomic bond energies. This is the same concept used above to estimate sublimation energies, rendering the following $E_{d}$ values: $E_{d}^{S i} \approx 19.5 \mathrm{eV}$ and $E_{d}^{N} \approx 14.6$ $\mathrm{eV}$. These $E_{d}$ values must be compared with the maximum energy $E_{\max }$ that can be transferred in a binary elastic 
TABLE II. Maximum energy transfer for electron-atom elastic collisions.

\begin{tabular}{|c|c|c|c|c|c|c|}
\hline \multirow[b]{2}{*}{$\begin{array}{l}E_{b} \\
(\mathrm{keV})\end{array}$} & \multicolumn{2}{|r|}{$\mathrm{N}$} & \multicolumn{2}{|r|}{$\mathrm{Si}$} & \multicolumn{2}{|r|}{$\mathrm{Au}$} \\
\hline & $\begin{array}{l}E_{\max } \\
(\mathrm{eV})\end{array}$ & $\begin{array}{c}E_{\max }-E_{d}^{N} \\
(\mathrm{eV})\end{array}$ & $\begin{array}{l}E_{\max } \\
(\mathrm{eV})\end{array}$ & $\begin{array}{c}E_{\max }-E_{d}^{S i} \\
(\mathrm{eV})\end{array}$ & $\begin{array}{l}E_{\max } \\
(\mathrm{eV})\end{array}$ & $\begin{array}{c}E_{\max }-E_{d}^{A u} \\
(\mathrm{eV})\end{array}$ \\
\hline 80 & 13.5 & -1.1 & 6.8 & -12.7 & 0.96 & $\ldots$ \\
\hline 120 & 21 & 6.4 & 10.5 & -9.0 & 1.5 & $\ldots$ \\
\hline 160 & 29 & 14.4 & 14.5 & -5.0 & 2.07 & $\ldots$ \\
\hline 200 & 37 & 22.4 & 18 & -1.5 & 2.67 & $\ldots$ \\
\hline
\end{tabular}

$E_{d}^{S i} \approx 19.5$ and $E_{d}^{N} \approx 14.6 \mathrm{eV}$.

electron-atom collision for $\mathrm{N}, \mathrm{Si}$, and $\mathrm{Au}$ atoms, as listed in Table II for the beam energies used in this work. According to Table II, fully coordinated $\mathrm{Si}$ atoms may not be directly displaced since $E_{\max }-E_{d}^{S i} \leq 0$, while the displacement of fully coordinated $\mathrm{N}$ atoms can only occur for energies $E_{b} \geq 120 \mathrm{keV}$. For $\mathrm{Au}$ atoms, however, it is difficult to predict their $E_{d}$ values. Assuming that they occupy interstitial positions, their displacement energy would depend on the potential energy barrier provided by the surrounding matrix atoms along the jump direction. Without this information, we may assume that, due to the small $E_{\max }$ values, direct displacements are quite improbable. Hence, the observed $\mathrm{Au}$ agglomeration within the slow growth regime can be best explained in terms of an indirect migration process, as suggested by Klimenkov et al. ${ }^{31}$ to explain the formation of $\mathrm{Ge}$ NPs in ion implanted silica films under electron irradiation at $200 \mathrm{keV}$. They argued that the displacement of $\mathrm{O}$ atoms modifies the local structure arrangement and induces the migration of $\mathrm{Ge}$ atoms. The same argument can be applied here with respect to the displacement of $\mathrm{N}$ atoms, and the indirect nature of the $\mathrm{Au}$ displacements may justify the observed slow agglomeration rate for $\Phi_{e}<\Phi_{e}^{i}$. In addition, we also speculate that, due to the implantation process of the $\mathrm{Au}$ atoms, the lattice damage caused by the displacements of $\mathrm{Si}$ and $\mathrm{N}$ atoms may not be fully recovered, giving rise to the possibility of a local weakening of the bound state of the matrix atoms, which would further contribute to the shortrange migration of the $\mathrm{Au}$ atoms.

For $\Phi_{e} \geq \Phi_{e}^{i}$, the situation is different. Here, Au clusters and individual $\mathrm{Au}$ atoms are located at the surface without forming strong covalent bonds with the matrix. In this case, the energy barrier constraint for atomic migration is rather small, thus rendering small $E_{d}$ values to promote the migration of $\mathrm{Au}$ atoms. On the basis of this concept, we will test whether elastic electron-atom collision events can trigger surface diffusion and eventually lead to the fast growth regime of the $\mathrm{Au}$ NPs. We also remark that, as irradiation proceeds, the $\mathrm{Au}$ atoms that remain inside the silicon nitride membrane may still contribute to the slow growth process. For irradiation fluences just overpassing the inflection $\Phi_{e}^{i}$ value, the surface located particles are quite small, and therefore, it is difficult to distinguish them from those located inside the membrane. Figures 3(a) and 3(b) are examples of this phenomenon. However, for higher fluences [see Figs. $3(\mathrm{c})$ and $3(\mathrm{~d})$ ], the micrographs reveal a bimodal particle size distribution, where the smaller ones are considered to still be inside the membrane. We assume, however, that most of the $\mathrm{Au}$ atoms inside the membrane are either still not attached to atomic clusters or that $\mathrm{Au}$ atoms can be easily detached from small atomic clusters. In this sense, Au atoms are continuously supplied to the surface via the electron irradiation induced thinning process. According to the present reasoning, the thinning rate does not change significantly with the beam energy, as suggested by the small difference in the $\Phi_{e}^{i}$ values. As a consequence, if direct displacements are the dominant process, then the distinct growth rates observed for the irradiations at 120, 160, and $200 \mathrm{keV}$ (see Fig. 6) should be proportional to the displacement crosssection $\sigma_{A u}\left(E_{e}, E_{d}\right)$ for the electron-atom collisions. As a first approach, we will assume that the growth process of the surface located particles follows a linear behavior given by

$$
d_{m}=d_{0}+k \cdot \sigma_{d}^{A u}\left(E_{e}, E_{d}\right) \cdot\left(\Phi_{e}-\Phi_{e}^{i}\right),
$$

where $d_{m}$ is the mean diameter of the Au particles, $d_{0}$ is the mean particle diameter at the inflection point, $k$ is a constant from the growth mechanism, $\sigma_{A u}\left(E_{e}, E_{d}\right)$ is the collision cross section, $\Phi_{e}$ is the electron irradiation fluence, and $\Phi_{e}^{i}$ is the fluence at the inflection point. The linear growth approach adopted in Eq. (3) results from the empirical observation of the data in Fig. 6. The linear behavior may result from the combination of two processes: (i) the supply of $\mathrm{Au}$ atoms and small particles because of the sputtering process taking place during the irradiation and (ii) an Ostwald ripening process induced by the irradiation. If the Au atoms were completely standing at the surface, a pure Ostwald ripening behavior with the growth exponent of 3 should be expected. ${ }^{32}$ According to Egerton et al. ${ }^{28}$ for heavy elements, elastic electron-atom collisions are best described by the displacement cross-section $\sigma_{A u}\left(E_{0}, E_{d}\right)$ calculated using the unscreened Rutherford approach, which reads

$$
\sigma^{u R}=\pi Z^{2} r_{0}^{2}\left[\left(1-\beta^{2}\right) / \beta^{4}\right]\left[\left(E_{\max } / E_{d}\right)-1\right] .
$$

In this equation, $\beta, E_{\max }$, and $E_{d}$ have the same meanings as in Eq. (2), $\mathrm{Z}$ is the atomic number, and $r_{0}=2.81794 \mathrm{fm}$ is the classical radius of the electron. Figure 9 shows the dependence of $\sigma_{A u}^{u R}$ as a function of $E_{e}$ for distinct $E_{d}$ values.

To test the consistency of Eq. (3), we empirically fit the data from the fast growth regime in Fig. 6, considering for all energy cases the same values of the two unknown parameters: the constant $k$ and displacement energy $E_{d}$ that sets the values of $\sigma_{A u}^{u R}$ as a function of the beam energy. The results obtained from the best fit approach (where $k$ $=2.218 \times 10^{6} \mathrm{~m} / \mathrm{C}$ and $E_{d}=1.25 \mathrm{eV}$ ) are shown as dotted lines in Fig. 6. It seems remarkable that Eq. (3) well describes the irradiation fluence behavior of the fast growth regime. If the present empirical approach holds, then the results suggest that the growth of the surface located Au NPs is indeed caused by atomic displacements produced by binary elastic collisions. The open circles in the $E_{d}=1.25$ $\mathrm{eV}$ curve in Fig. 9 represent the $\sigma_{A u}^{u R}$ values used in the present linear approach describing the slope of the fast growth regime. In addition, the $\sigma_{A u}^{u R}$ value for $E_{d}=1.25 \mathrm{eV}$ vanishes 

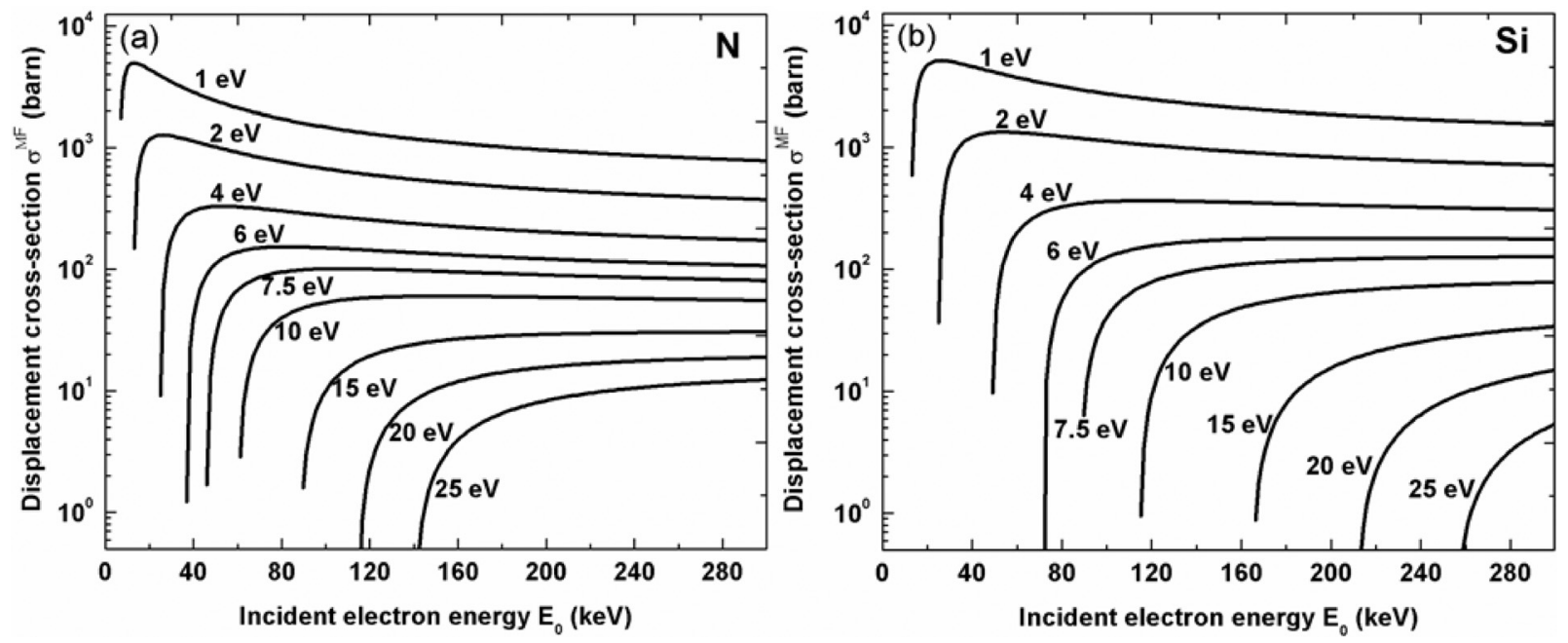

FIG. 8. McKinley-Feshbach approximation for the elastic displacement cross-sections $\sigma^{M F}$ : (a) $\sigma_{N}^{M F}$ and (b) $\sigma_{S i}^{M F}$ plots as a function of the incident electron energy at $E_{e}$. Each curve corresponds to a distinct $E_{d}$ value indicated in the curve label.

for smaller beam energies, consistently explaining why the fast growth regime did not occur for the $80 \mathrm{kV}$ irradiation case.

If the present analysis is correct, it suggests that the NP growth process can be promoted via the surface migration of $\mathrm{Au}$ atoms induced by binary elastic electron-atom collisions with a rather low displacement energy $\left(E_{d}=1.25 \pm 0.25 \mathrm{eV}\right)$ compared with similar data from the literature. For example, Lee and Mori ${ }^{33}$ have reported that $\mathrm{Au}$ atoms were selectively removed from $\mathrm{Al}_{2} \mathrm{Au}$ NPs during irradiation experiments at $150 \mathrm{keV}$, estimating a displacement energy $E_{d} \approx 1.9 \mathrm{eV}$. Furthermore, Ma and Marks ${ }^{34}$ reported that the diffusion of $\mathrm{Au}$ atoms in a free Au crystal surface could be induced by electrons accelerated at $135 \mathrm{keV}$. Last, but not least, Gan et $a l .{ }^{35}$ suggested that the activation energy for surface diffusion of $\mathrm{Au}$ atoms in graphene corresponds to $\approx 2.3 \mathrm{eV}$, which can be transferred by elastic collisions with beam energies $E_{b}>170 \mathrm{keV}$. In this sense, we may remark that our observations report on a rather complex phenomenon where the migration of $\mathrm{Au}$ atoms occurs concomitantly with

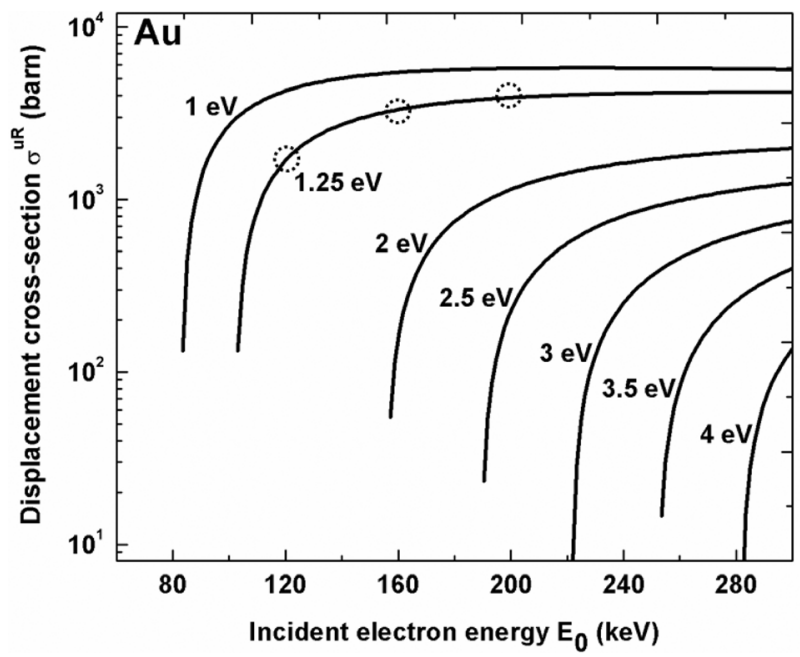

FIG. 9. Unscreened Rutherford displacement cross-section for Au as a function of the incident electron energy $E_{0}$ for different values of displacement energy $E_{d}$. the sputtering of $\mathrm{Si}$ and $\mathrm{N}$ atoms. Hence, the displacement of $\mathrm{Si}$ and $\mathrm{N}$ atoms may also indirectly enhance the Au migration process, thus rendering an apparent lower displacement energy for the $\mathrm{Au}$ atoms if only direct knock-on collisions are taken into account.

Notwithstanding, the phenomenon of Au agglomeration via electron irradiation can be characterized as a physical tool to promote the configuration of organized nanostructures which avoids thermal effects. To further demonstrate the potential of the method, we performed agglomeration experiments using the scanning transmission electron microscopy (STEM) mode with an electron probe of $\approx 1 \mathrm{~nm}$ from a probe corrected microscope operating at $300 \mathrm{kV}$. The results obtained are shown in Fig. 10. This figure demonstrates that $\mathrm{Au}$ nanostructures (rods and dots), even presenting subnanometric sizes, can be produced at the desired locations, thus suggesting that the present electron-beam induced agglomeration technique can be used as a fine tool to produce welllocalized atomic clusters or nanoparticles.

\section{CONCLUSIONS}

In the present contribution, we studied the thermal and electron irradiation effects on silicon nitride membranes and

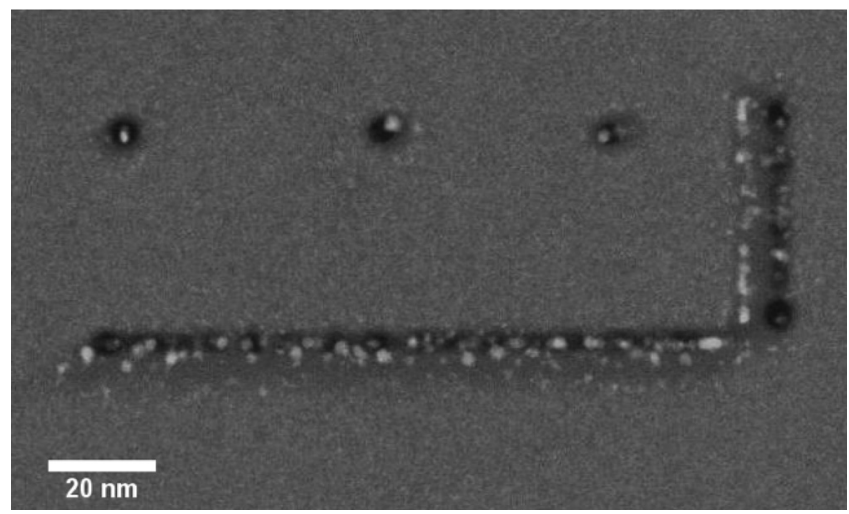

FIG. 10. Z-contrast TEM micrograph of an Au nanoparticle system produced by $300 \mathrm{keV}$ electron irradiation using a $1 \mathrm{~nm}$ wide electron probe. 
thin films ion implanted with Au. We demonstrated that the system is rather robust with respect to thermal annealing at temperatures up to $1100^{\circ} \mathrm{C}$ but is quite sensitive to room temperature electron irradiation effects performed with beam energies from 80 to $200 \mathrm{keV}$.

The results obtained show that, concomitantly with the sample thinning, the formation of Au nanoparticles occurs, with the growth rates increasing with the beam energy. The thinning process was analyzed in terms of atomic sputtering directly caused by electron-atom binary elastic collisions. Calculations based on realistic displacement cross-sections render surface displacement energies for $\mathrm{Si}$ and $\mathrm{N}$ atoms around $14 \pm 3 \mathrm{eV}$. These energies can be underestimated if additional thinning processes, such as a net atomic migration toward the boundaries of the irradiated area, are operative.

The growth of Au nanoparticles was discussed in terms of two growth regimes. The first regime presents a small growth rate, assuming that the Au migration is indirectly caused by the displacement of $\mathrm{N}$ atoms. The second regime is characterized by a larger growth rate and begins at a welldefined fluence value, where the sample thinning process finally exposes Au atoms to the sample back surface. The results are analyzed in terms of electron-induced atomic displacements simultaneously, causing the thinning of the silicon nitride membrane and therefore supplying $\mathrm{Au}$ atoms to the surface, and the migration of the surface located $\mathrm{Au}$ atoms, causing the growth of Au nanoparticles. An analysis considering purely ballistic induced displacements also based on a binary elastic collision approach is performed. This analysis considers realistic displacement cross-sections and suggests that the Au nanoparticle growth rate is directly proportional to the displacement cross-section of $\mathrm{Au}$ atoms with a surface displacement energy of $E_{d} \approx 1.25 \pm 0.2 \mathrm{eV}$. This energy may also be underestimated if the Au surface migration is enhanced by the $\mathrm{Si}$ and $\mathrm{N}$ displacement processes.

The formation of $\mathrm{Au}$ nanostructures is additionally explored using a $1 \mathrm{~nm}$ wide electron probe, demonstrating that even subnanometric particles can be formed in welldefined positions, thus characterizing focused electron beam irradiations as a promising tool for tailoring the formation of nanoparticles with well-defined sizes and locations.

\section{ACKNOWLEDGMENTS}

The authors would like to acknowledge the financial support from the Brazilian Agencies CNPq and CAPES.

The authors declare that they have no conflict of interest with respect to funding sources or authorship.
${ }^{1}$ Q. S. Mei and K. Lu, Prog. Mater. Sci. 52, 1175-1262 (2007).

${ }^{2}$ A. Aguado and M. F. Jarrold, Annu. Rev. Phys. Chem. 62, 151-172 (2011).

${ }^{3}$ F. Ruffino, M. G. Grimaldi, F. Giannazzo, F. Roccaforte, and V. Raineri, Appl. Phys. Lett. 89, 263108 (2006).

${ }^{4}$ F. Ruffino, M. G. Grimaldi, F. Giannazzo, F. Roccaforte, and V. Raineri, Appl. Phys. Lett. 89, 243113 (2006).

${ }^{5}$ H. Häkkinen, Chem. Soc. Rev. 37, 1847 (2008).

${ }^{6}$ K. L. Kelly, E. Coronado, L. Zhao, and G. C. Schatz, J. Phys. Chem. B 107, 668-677 (2003).

${ }^{7}$ B. Roldan and F. Behafarid, Surf. Sci. Rep. 70, 135-187 (2015).

${ }^{8}$ K. Dick, T. Dhanasekaran, Z. Zhang, and D. Meisel, J. Am. Chem. Soc. 124, 2312-2317 (2002)

${ }^{9}$ A. Safaei and M. Attarian Shandiz, Phys. Chem. Chem. Phys. 12, 15372-15381 (2010).

${ }^{10}$ F. Kremer, J. M. J. Lopes, F. C. Zawislak, and P. F. P. Fichtner, Appl. Phys. Lett. 91, 083102 (2007).

${ }^{11}$ F. P. Luce, F. Kremer, S. Reboh, Z. E. Fabrim, D. F. Sanchez, F. C. Zawislak, and P. F. P. Fichtner, J. Appl. Phys. 109, 014320 (2011).

${ }^{12}$ C. Q. Sun, C. M. Li, H. L. Bai, and E. Y. Jiang, Nanotechnology 16, 1290-1293 (2005).

${ }^{13}$ D. A. Porter, Phase Transformations in Metals and Alloys (CRC Press, New York, 2009).

${ }^{14}$ P. W. Voorhees, J. Stat. Phys. 38, 231-252 (1985).

${ }^{15}$ M. Zinke-Allmang, L. C. Feldman, and M. H. Grabow, Surf. Sci. Rep. 16, 377-463 (1992).

${ }^{16}$ F. P. Luce, E. Oliviero, G. D. Azevedo, D. L. Baptista, F. C. Zawislak, and P. F. P. Fichtner, J. Appl. Phys. 119, 035901 (2016).

${ }^{17}$ W.-K. Chu, J. W. Mayer, and M.-A. Nicolet, Backscattering Spectrometry (Academic Press, Inc., New York, 1978).

${ }^{18}$ F. Banhart, Rep. Prog. Phys. 62, 1181-1221 (1999).

${ }^{19}$ O. Ugurlu, J. Haus, A. A. Gunawan, M. G. Thomas, S. Maheshwari, M. Tsapatsis, and K. A. Mkhoyan, Phys. Rev. B 83, 113408 (2011).

${ }^{20}$ T. Yokota, M. Murayama, and J. M. Howe, Phys. Rev. Lett. 91, 265504 (2003).

${ }^{21}$ G. S. Was, Fundamentals of Radiation Materials Science (Springer, 2007).

${ }^{22}$ P. A. Crozier, M. R. McCartney, and D. J. Smith, Surf. Sci. 237, 232-240 (1990).

${ }^{23}$ R. F. Egerton, P. Li, and M. Malac, Micron 35, 399-409 (2004).

${ }^{24}$ R. F. Egerton, F. Wang, and P. A. Crozier, Microsc. Microanal. 12, 65-71 (2006).

${ }^{25}$ D. G. Howitt, S. J. Chen, B. C. Gierhart, R. L. Smith, and S. D. Collins, J. Appl. Phys. 103, 024310 (2008).

${ }^{26}$ A. Zobelli, A. Gloter, C. P. Ewels, G. Seifert, and C. Colliex, Phys. Rev. B 75, 245402 (2007).

${ }^{27}$ K. A. Mkhoyan and J. Silcox, Appl. Phys. Lett. 82, 859-861 (2003).

${ }^{28}$ R. F. Egerton, R. McLeod, F. Wang, and M. Malac, Ultramicroscopy 110, 991-997 (2010).

${ }^{29}$ D. R. Lide, Handbook of Chemistry and Physics (CRC Press, 2004).

${ }^{30}$ J. F. Ziegler and J. P. Biersack (2003), see http://www.srim.org/ to download the SRIM code and obtain information about displacement energies.

${ }^{31}$ M. Klimenkov, W. Matz, and J. V. Borany, Nucl. Instrum. Methods Phys. Res., Sect. B 168, 367-374 (2000).

${ }^{32}$ F. Ruffino, M. G. Grimaldi, F. Giannazzo, F. Roccaforte, V. Raineri, C. Bongiorno, and C. Spinella, J. Phys. D: Appl. Phys. 42, 75304 (2009).

${ }^{33}$ J. G. Lee and H. Mori, Sci. Technol. Adv. Mater. 5, 51-55 (2004).

${ }^{34}$ Y. Ma and L. D. Marks, Ultramicroscopy 31, 193-197 (1989).

${ }^{35}$ Y. Gan, L. Sun, and F. Banhart, Small 4, 587-591 (2008). 